\title{
Multistage Processes of Identifying Children at Risk or Out of Family Care: a Case of DOVCU Project Methods in Uganda
}

\author{
Fred Mutenyo ${ }^{1} \cdot$ Simba Machingaidze ${ }^{1} \cdot$ Walter Okello $^{1}$ (D) $\cdot$ Moses Otai $^{2} \cdot$ Monica Asekenye $^{2}$
}

Published online: 14 February 2019

(C) The Author(s) 2019

\begin{abstract}
By 1996, Uganda had about 36 Child Care Institutions (CCIs), also known as orphanages. Over recent years, there was an exponential increase in number of CCIs and most of them are not formally licensed by the government. Available evidence in Uganda indicates that institutional care is taken as priority response to out of family children without exploring family-based care options and this has detrimental effects on child development and wellbeing as well as the future. Key factors such as HIV and AIDS, child abuse and neglect, endemic poverty, migration, and family breakdown have contributed to the increase in institutionalization of children. It is against this backdrop that the Uganda Government through MGLSD introduced a National Framework for Alternative Care in the year 2012, in line with the UN Alternative Care Guidelines and Article 20(3) of the UN Convention on the Rights of the Child (CRC) that emphasized institutional care as a last resort. In this context, and the subsequent domestication of the UN guidelines, coupled with evidence of an unregulated increase in the use of residential care for orphans and vulnerable children in Uganda, the Deinstitutionalization of Orphans and Vulnerable Children in Uganda (DOVCU) project was very relevant and timely. The overall objective of the project was to support actualization of Alternative Care Framework (ACF) in Uganda, by working with CCIs to reconceptualize and embrace family-based care but also work with communities to prevent separation of children. To achieve the above objectives, it was imperative to design a clear methodological protocol to (1) identify districts with highest numbers of CCIs, (2) assess CCIs conformity to the national approved home regulation standards, (3) assess all children in the CCIs to identify common reasons for their admission in the care institutions, (4) identify children with case plan including those who wish to be reunified in family, and (5) identify communities that supply most record number of children to the CCIs. This paper also articulates key steps in which other processes were to be undertaken: (1) to identify common push and pull factors to family-child separation based on various community context, (2) to identify households at risk of child separation based on community knowledge, (3) to assess vulnerability levels among both at risk of separation and already separated households and their children, (4) to classify household vulnerability levels based on the tested vulnerability index tools, and (5) to appropriately target vulnerable households with integrated intervention packages which were mainly cash transfers and support to improve household access to financial capital and credit. In addition, the paper presents a longitudinal process through which the project participants were monitored along the vulnerability scales from baseline, through wave three of data collection, where evidences from DOVCU integrated package of services provided useful learning to practitioners in OVC programs.
\end{abstract}

Keywords Deinstitutionalization - Orphans and vulnerable children · Child care institutions - Alternative Care Framework . Participatory rural appraisal $\cdot$ Household and child vulnerability assessments tool $\cdot$ Reintegrating and prevention households

Walter Okello

wokello@ childfund.org

Fred Mutenyo

fmutenyo@childfund.org

Simba Machingaidze

smachingaidze@childfund.org

Moses Otai

motai@childfund.org
Monica Asekenye

masekenye@childfund.org

1 Department of Monitoring and Evaluation, ChildFund International Uganda, Plot 71/72 Namirembe Road-Bakuli, Kampala, Uganda

2 Department of Programs, ChildFund International Uganda, Plot 71/ 72 Namirembe Road-Bakuli, Kampala, Uganda 


\section{Introduction and Background}

In 1996, Uganda had approximately 36 residential Child Care Institutions (CCIs) also known as orphanages. Currently, there is an estimated $800 \mathrm{CCIs}$, with approximately 150,000 children, worth noting is that only 70 institutions are licensed by the Ugandan Ministry of Gender, Labour and Social Development (MGLSD 2012). Some institutions operate without licenses, others disguise as a school, nongovernment organization (NGO), and faith-based organization (FBOs) and the status of others are not known to both MGLSD and the various districts. Institutionalization of children in Uganda is a reality. Available evidence indicates that institutional care is used often as a "first resort" response without consideration for or investment in family-based care options (MGLSD 2012). Since institutionalization of children does not ensure the best interest of children and has a negative impact on their development, wellbeing, and future, it is important to provide care and protection for them within a family environment in all situations possible. However, the increase in institutional care has coincided with increasing awareness of and research into the negative effects of institutionalization. Children in institutional care are particularly vulnerable needing special care and protection (Tolfree 1995). They are entitled to an environment that guarantees them protection from all forms of violence. Despite the existence of rights, children suffer from poverty, homelessness, abuse, neglect, mental and physical diseases, and unequal access to education and justice systems that do not recognize their special needs.

The issues pertaining to institutionalized children and their vulnerability are very complex. Increasing physical and mental maturity usually leads to growing capability for self-provisioning, but during periods of childhood and adolescence, children and young people continue to need special care and support (Bee and Boyd 2004). The institutionalization can be an outcome of many factors such as poverty, ill health, and/or social exclusion. While according to studies, economic factors have mainly influenced institutionalization, and social deprivation in all forms has been identified as the major underlying factor for institutionalization of children. Here is a myriad of research over several decades that demonstrate the negative effects of children growing up in institutions; numerous studies have provided convincing evidence on the fact that institutional care is detrimental to the cognitive, behavioral, emotional, and social development of young children (Galappatti 2002; Parry-Williams 2006; Roccella 2007; Priyangani et al. 2005; United Nations 2009). It is well documented that children who are institutionalized during the first 6 months of their lives suffer long-term developmental delay, leading to a greater probability of antisocial behavior and mental health problems (UNICEF 2010).
A number of observational studies have compared institutionalized children to non-institutionalized children. These studies tell a compelling story of the effects of institutional care; most studies find that institutionalized children have significant developmental deficits across virtually every domain that has been examined (e.g., Guinnar, Van Dulmen, and The International Adoption Project Team, 2007; Kreppner, O'Connor, and Rutter, 2001; van IJzendoorn, Luijk, and Juffer, 2008). Children living in institutional care show very significant deficits in intellectual and cognitive development (Carlson and Earls 1997; Johnson et al., 1996; Kreppner, O'Connor, and Rutter, 2001; Moulson, Westerlund, Fox, Zeanah, and Nelson, 2009; O'Connor et al., 2000; Smyke et al., 2007). Children in institutional care lag behind other children in physical development. Relative to their peers, they show a typically short height, low weight, and small head circumference (Johnson et al., 1996; Smyke et al., 2007). Smyke et al. (2007) found that height, weight, and head circumference of infants and toddlers in institutions was about a standard deviation below norms and significantly different from children living in the community. Johnson et al. (1996) found that children in Romania lost about 1 month of growth for every 3 months of institutional care, whereas children in the former Soviet Union showed 1 month growth delay for every 5 months of institutional care. A meta-analysis conducted by van IJzendoorn, Bakermans-Kranenburg, and Juffer (2007) found that the longer infants remained in institutional care, the more they differed from normal growth parameters. Zeanah et al. (2005) found that $44 \%$ of institutionalized children showed high levels of indiscriminately sociable behavior as contrasted with $18 \%$ of children who had never been institutionalized.

Institutionalization has also contributed to the neglect of individual and personal care and provides limited stimulation for children to respond effectively to their psychological needs (De Silva and Punchihewa 2011). According UNICEF (2009), children in institutional care constitute one of the most marginalized, underserved, and most vulnerable group of children. The effects of institutionalization on child health, development, and wellbeing as illustrated in several studies have not been encouraging (De Silva and Punchihewa 2011).

Furthermore, institutionalization of children involves making children reside in orphanages or CCIs, rather than with their biological families, in kinship families, living with a foster family or adoptive family (Uganda Alternative Care framework 2012). Children in institutional care are high risks of abuse, neglect, exploitation, and manipulation by those should to protect (Colton 2002 and Sellick 1998). As children stay in the institutions, they are often deprived family bonding with their parents or kinship members of the families. In some instances, they are exposed to unhealthy living conditions and harsh disciplinary measures by the 
care takers in the institutions. Indeed, research is increasingly pointing to negative effects of institutionalization on children's physical, emotional, and cognitive development (Browne 2009; Csaky 2009; Johnson and Gunnar 2011). Inspirit of the detrimental effects of institutionalization, there are a number of push factors that lead children to institutionalized care.

In Uganda, key factors such as HIV and AIDS, child abuse and neglect, endemic poverty, migration, and family breakdown have contributed to the increase in the number of children requiring alternative care (MGLSD 2010, 2012; Walakira et al. 2014a). Children are furthermore exposed to a multitude of protection risks. Child marriage is very prevalent, approximately $25 \%$ of children are estimated to be engaged in child labor (UNICEF 2015), and an estimated 10,000 children are thought to be living on the street. Levels of violence against children are also high (Walakira et al. (2014b)). Children's care and living arrangements are also a reflection of child vulnerabilities in Uganda. According to Better Care Network and UNICEF (2009), only $56 \%$ of children under the age of 18 are living with both biological parents.

Against that backdrop, the Government of Uganda (2012) introduced a National Framework for Alternative Care in the year 2012. This framework is in line with the UN Alternative Care Guidelines and Article 20(3) of the UN Convention on the Rights of the Child (CRC) that calls for institutional care to be a last resort for children in need of alternative care once all other care options along the continuum, namely, family reunification, kinship and community care, domestic adoption, foster care, and inter-country adoption have been exhausted (MGLSD 2011). In this context, following the adoption of the UN Guidelines on Alternative Care at a global level in 2009, and the subsequent domestication of these guidelines in the national Alternative Care Framework in 2011, coupled with evidence of an unregulated increase in the use of residential care for orphans and vulnerable children in Uganda, the DOVCU project was very relevant, timely and needed to contribute to efforts in deinstitutionalization of orphans and vulnerable children in Uganda.

The Deinstitutionalization of Orphans and Vulnerable Children in Uganda (DOVCU) project 2014-2017 funded by USAID aimed at measurably improving the safety, wellbeing, and development of highly vulnerable children, both those at risk of family separation and children living outside familybased care across targeted districts in Uganda, specifically:

1. To reduce unnecessary separation of children from families

2. To place children outside family care in nurturing families

\section{Rationale of the Methodology}

To achieve the above two objectives, the DOVCU project aimed at strengthening household livelihood, promoting positive parenting, strengthening PSS, awareness creation on child protection, and supporting Government of Uganda efforts to implement the Alternative Care Framework (ACF) as well as strengthening human development and institutional capacities at family, community, districts and national level. The project was also expected to contribute to the growing evidenceslinking integration of economic strengthening and social interventions to positive outcomes for children and their families. In a bid to sequentially deliver the project intervention packages with evidences, it was imperative to design a clear systematic qualitative and quantitative methodology that would be followed at every level of implementation:

- To identify the districts, child care institutions and Remand Homes (RH), and specific communities to work with

- To use the community knowledge to identify the push and pull factors driving children to the CCIs, streets, or commit crime and be reprimanded

- To engage key stakeholders at the community level through the PRA/community reflections to identify households where children were at risk of separating from their families

- To examine the vulnerability status of the identified families and their children through a household level assessment using the Family Status Vulnerability Index (FSVI) tool and Child Status Vulnerability Index (CSI) tool

This paper therefore articulates the logical steps that was undertaken to identify districts, CCIs, RH, sub-counties, and parishes to work with. It also seeks to categorically outline the inclusive process that was used to examine push and pull factors of family-child separation, identify households at risk of family-child separation "prevention households," identify reunifying children and trace their households "reintegrating households," and assess and classify in quantified terms the level of vulnerability in both at risk and separated households.

\section{Systematic Assessment Process}

(a) As fully illustrated in the Fig 1, first was to select the project implementation areas using a study conducted by the Ministry of Gender, Labour and Social Development (MGLSD) in 2014 that indicated districts with highest number of CCIs in Uganda. The report indicated that 19 of the CCIs were in the Northern region, 61 in Eastern, 18 in Western, and 203 in central Uganda (MGLSD, 2014). The DOVCU project based on this to select the top 12 districts to implement its interventions. The 12 districts were spread across the four regions of Uganda that included Northern: Gulu and Lira districts; Eastern: Mbale, Jinja, Iganga, and Kamuli districts; 
Fig. 1 DOVCU project systematic roll-out process

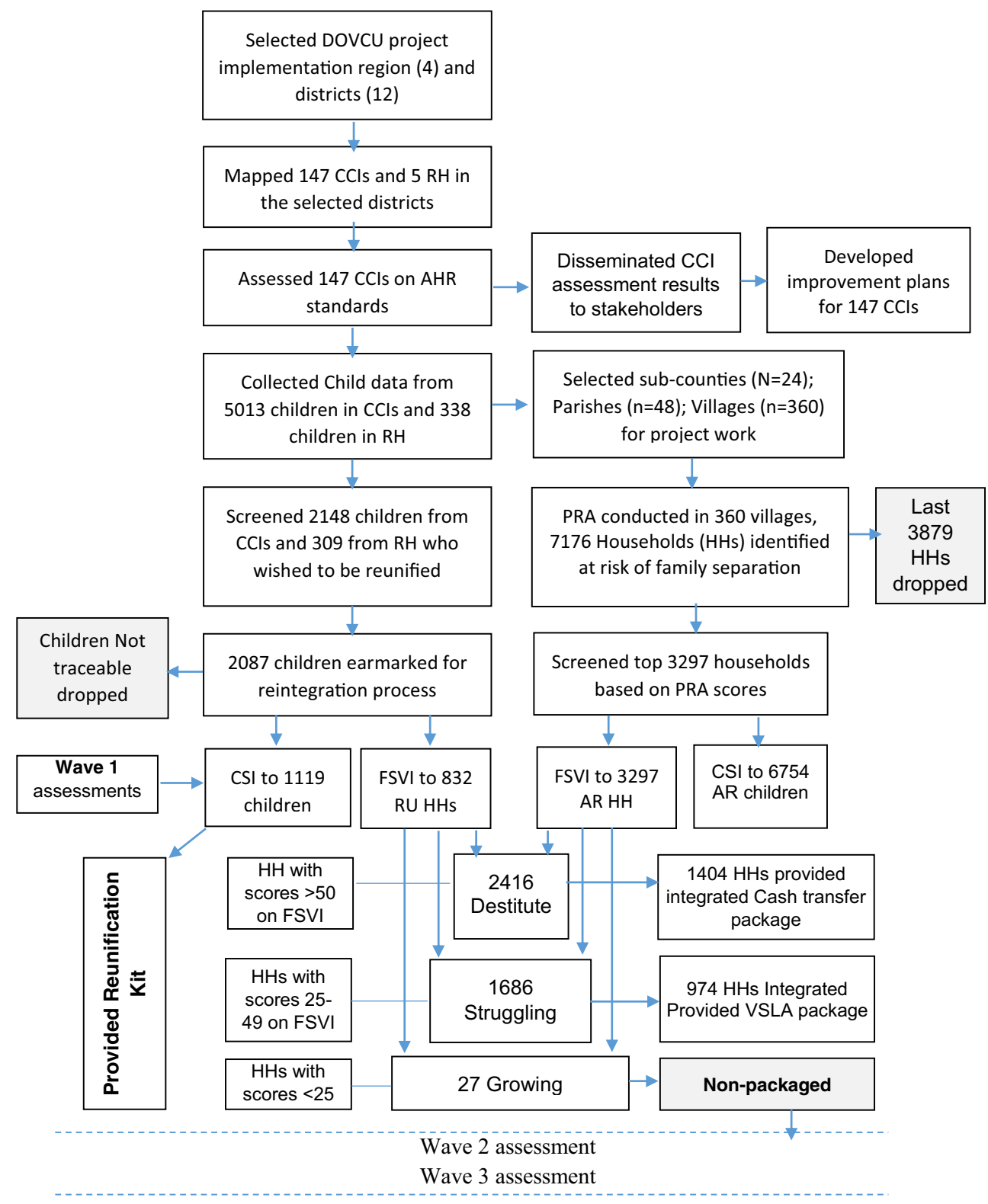

Western: Kaesese, Kaborale, and Kabale districts; and Central Uganda: Waksio, Kampala, and Luwero districts.

A list of CCIs within the 12 districts were obtained from the respective District Community Service Department at the district and sub-county level, and the project targeted to work with all the CCIs in 10 districts, except Kampala and Wakiso districts where CCIs were selected based on their location within the project-targeted divisions, but also targeting those that most needed improvement on approved home regulation standards. A total of 147 CCIs mapped were earmarked to implement the ACF under DOVCU support. The CCIs were then visited by the assessment team that comprised of representatives from the MGLSD, District Health Department, Education Department, Production Department, and Community Service Department and representatives from the Police Child and Family Protection Unit (PCFPU) across the respective districts.

(b) The second step was to evaluate the conformity and performance of the CCIs on the Approved Home Regulation (AHR) standards. To do this a MGLSD CCI assessment tool kit designed in accordance with Children Approved Homes Rules (AHR) 2013 was used. This AHR guideline outlines the key ideals that need to be put in place for an institution to be rated as in conformity to the approved home regulation standards and to also operate as a CCI/or an orphanage. The eight areas/parameters in the assessment tool kit included:

1. Governance and management structure

2. Financial management systems 
3. Presence of inspection and reports from the Ministry of Gender, Labour and Social Development (MGLSD) and probation and social welfare officer (PSWO) as the mandated supervisory bodies

4. Human resource policies and case-loads for staff

5. Child care provisions

6. Record keeping systems

7. Child resettlement and alternative care

8. Post placement support for children that are reintegrated with nurturing families

Each parameter had a score range of 0 to $4-$ where zero implied given standards were not in place or not considered, completely inadequate, or non-compliance with legislation, regulation, and best practices; one implied considered/planned but not implemented, severely deficient; two implied partially implemented or informally or undocumented or even lacking; three implied implemented but undefined/undocumented or needs some additional work or modification; and four implied the standards were fully implemented/complied fully with legislation, regulation, and best practice/well documented and reviewed frequently. For each of the parameters, there were different number of questions, and each of the questions was scored in the range 0-4 appropriately. Later, the assessment team would get sum of all items (questions) and divide by number of questions to obtain the CCI average score for each parameter (standards). As mentioned earlier, the CCI average score ranged between 0 and 4 , from on this assessment, action recommendations for each of the CCIs were drawn based on two key dimensions:

1. The CCI average score on all the standards

2. Observations made by the assessment team on issues regarding child friendliness of the home and safe environment including the hygiene and sanitation within the CCI (orphanage)

The possible recommended actions for each CCI were immediate closure, closure in 3 months, closure in 6 months, recommended improvement, further investigation, and recommend for approval. CCIs that were recommended for improvement were supposed to improve on the parameters in which they were scored low; care institutions who were recommended for further investigation required revisits by the assessment team. Institutions that were recommended for approval were to formalize their operation by applying for registration as CCIs to the MoGLSD. The results of the CCI assessments were shared with the MGLSD including the respective district PSWO who are the mandated government entities to enforce the Alternative Care (ATC) policy.
Irrespective of the action recommendations and government follow-up on the recommendations, CCI assessment results offered the Project Senior Social Workers and Program Officers a platform to draw improvement plans for all the 147 CCIs assessed on the AHR standards. Continuous CCI follow-up (monitoring) assessments were conducted in two waves of data collection, at midterm and end line.

(c) The third step was to ensure conformity to ethical standards and scientific merit of research involving human subjects; the DOVCU project proposal, evaluation design protocol, consent forms, and data collection tools were submitted to the Mildmay Uganda Research and Ethics Committee for review, guidance, and approval before individual-level interview process during the inception phase.

(d) After the ethical approval, enumerators were hired and trained on research ethics and the child data capture tools were translated in the various local languages; data was collected from all children (5013) in residential within the targeted 147 CCIs. Additionally, 338 children in contact with the law in the targeted (5) remand homes were also examined. The child data collection tool was intended to capture the following basic information: CCI location, child bio data, location of the child's origin, circumstances that led to admission in the CCI or crime committed that led to the arrest, parental status of the child, child's wishes (opinion) about family reunification, and some of the protection concerns experienced by the children within the CCIs.

(e) The child data results later revealed that $43 \%$ (2148) of children in the 147 targeted CCIs wished to be reunified with their family. Ninety-four percent (2023) of them had records or knew the name of the adult they were living before separation, 94\% (2026) knew their district of origin, and 86\% (1852) knew their particular sub-county of location before separation; this information indicated that tracing and reunification processes were more feasible for children who knew their location of origin before separation. In the remand homes, $91 \%$ (309) of the children wished to be reunified with family, $89 \%$ (298) of them knew the name of the adult they were living before being arrested, $87 \%$ knew their district of origin, and 59\% (295) knew their particular sub-county of location before the arrest. Based on these statistics, the DOVCU project then targeted to reunify $85 \%$ (2087) of the 2457 children from both CCIs and remand homes who had case plans and wished to be reunified with their families. About $16 \%$ of children in the CCIs and remand home who wished to be reunified were from Wakiso and Kampala district alone, and this finding necessitated the DOVCU project to earmark one of the two districts to implement only objective 2 of the project - to place children outside family care in nurturing families. Statistics on the name and location of the adult a child was living with before separation was very helpful to case managers in preparation of further case plans for each child, family tracing, prereunification family preparation, and resettlement process. 
By end line, 1743 children were directly and indirectly provided alternative care options, and $99 \%$ were reunified with their biological families.

(f) Using the same child data from the $147 \mathrm{CCIs}$ and 5 remand homes, top districts supplying most record number of children to the CCIs and remand homes were established by ranking the number of children in the CCIs by their district of origin before separation. This data confirmed that the largest number of children in residential care originated from the very top 12 districts hosting the highest number of CCIs including those with remand homes. The next level of data analysis was to determine from within the top 12 districts, which sub-counties supplied the largest number of children in the CCIs and remand homes. Through a ranking method, top 2 sub-counties and top 2 parishes from within each of the top 2 sub-counties were selected as the intervention areas for DOVCU, especially for implementation of objective one of the project - to reduce unnecessary separation of children from families. Through this systematic data-driven process, the DOVCU project then worked in 24 sub-counties, 48 parishes and 360 villages within the 12 districts known as the "CCI and remand home supply communities." Feedback and data validation workshops were held with the district stakeholders to triangulate their informed position and the findings obtained using the CCI and remand home child statistics. This validation process was undertaken to ensure that the identification of the most burdensome top supply districts, sub-counties, and parishes was as inclusive as possible.

(g) The next stage was to engage community members from the 360 villages within the 48 parishes to assess unique priority risk factors (PRFs) in each community that explains drivers of family-child separation. Using the PRA approach, the project worked with community leaders to mobilize members for community reflection meetings in all the 360 villages, the rationale was to examine unique priority risk factors for family-child separation in each village to inform community own implementation plan to reduce unnecessary family-child separation. With help of the same enumerators (8-12) used in the CCI and remand home child data collection, 360 PRA sessions were executed using the following steps:

1. The community local council one chairperson in each village was facilitated to mobilize between 20 and 30 community knowledgeable participants in a common meeting place; this followed a well-laid data collection plan developed by case managers (Program Officers) who were based in the respective districts, Participants mobilized for the community PRA sessions were representatives of youth, female, male, opinion leaders, traditional leaders, elders, and people with disability $(\mathrm{PwD})$ and including a good geographical representation of people from all corners of each village.
2. Each PRA session commenced by briefing the participants on the DOVCU project, its objectives, and how community participation is being considered critical in the project implementation processes. Community members were asked to give their perspective of factors that lead to family-child separation or attract children to go: (a) on the street, (b) be admitted in CCIs, or (c) commit crime and end up being reprimanded. Participants exhaustively listed all the known factors that have led and still continuing to drive children outside family care in their community context. Data was captured in note books by note takers and on the flip chart by the facilitator. Each of the Priority Risk Factors (PRFs) was assigned a symbol drawn on a manila paper spread in front of the gathering or pinned on the wall, tree, depending on the nature of the PRA workshop venue; this was to ease communication with those who could not read and write.

3. DOVCU project intent was to select the six most prominent push and pull factors that lead to family-child separation in each community context. Using the exhaustive list of both pull and push factors, participants were then guided to rank the six most common priority factors that the community considers as the most critical factors. Using a proportionate piling exercise, the top 6 PRFs in each of the 360 villages were determined by ranking in descending order of piles stacked for each of the enlisted factors. The top six factors varied from one village to another, basing on each individual community context.

Participants then discussed how each of the top 6 PRFs leads to family-child separation in their own context, and verbatim notes were being captured by the note taker as the discussions brew with facilitators guide. Discussions continued, as the co-facilitator prepares PRA score table for the community PRA workshop with the guide from DOVCU project PRA protocol. The top 6 PRFs were assigned scores as follows: PRF 1 was assigned the highest PRA score of 5, PRF 2 was assigned a score of 4 , and PRF 6 was assigned a score of 0 , in that order as displayed in Table 1 below. The weight of 5 , $4,3,2,1$, and 0 was designed to give the highest weight of 5 to the top PRF and the weight of 0 to the least PRF based on the community ranking. Participants were not provided the logic behind the computation of the score and the order of the PRF, this was deigned to control for biases.

4. Through participatory discussions, participants were guided to reflect and recall of household(s) with children between the age of 0 and 17 within their surroundings or neighborhoods who identify with some if not all the top 6 PRFs. At this stage, each participant was free to mention a specific household (by name of the household head) they think was at risk or have already separated with a child or children in the most recent past. 
Table 1 PRA score guide

\begin{tabular}{ll}
\hline Community identified PRF & PRA score \\
\hline Priority risk 1 & 5 \\
Priority risk 2 & 4 \\
Priority risk 3 & 3 \\
Priority risk 4 & 2 \\
Priority risk 5 & 1 \\
Priority risk 6 & 0 \\
\hline
\end{tabular}

Source: DOVCU project protocol design

Depending on the type of risk factor, some of which were stigmatizing, participants were also free to secretly provide the households' detail to the co-facilitator and note taker with support from a Community Resource Persons (CORPs) that included Local Councils, alternatively VHTs. The at-risk households were captured by the names of the family head as indicated in Fig. 2 below; a scanned sample of completed At Risk of Child Separation Household Identification (ARCHI) card. In the end, a total of 7176 at risk of familychild separation households were identified.

Based on this tool, the maximum sum of scores attainable for a household was 15 points, which meant such as a household identified with all the top 5 PRFs. The least sum of score was zero, and such a household identified with only one PRFs and that was the sixth PRF.

Using DOVCU PRA guideline, each household score was added up; households with total PRA score of 10 and beyond were classified as at high risk (HR), households with total PRA scores ranging between 5 and 9 were classified as at medium risk, and those with total PRA score ranging between 0 and 4 were classified as at low-risk of
Table 2 Pre-selection risk analysis guide

\begin{tabular}{ll}
\hline Sum of PRA score & Type of risk \\
\hline $10+$ & High risk \\
$5-9$ & Medium \\
$0-4$ & Low risk \\
\hline Source: DOVCU project protocol design
\end{tabular}

Source: DOVCU project protocol design

family-child separation as shown in Table 2 below, risk classification computation criteria were not declared to the participants in order to control for biases during enlisting of at risk of separation households.

Using the information above, DOVCU project targeted strictly households classified at high and medium risk of separation, and those classified at low risk were dropped. With help of the CORPs in each village where the PRA took place, a sketch map of the village was drawn on a manila paper, with major features such as main roads, schools, streams, boreholes, and market places later plotted the location of all households at high and medium risk using the name of household head as captured in the ARCHI card.

The high- and medium-risk households were earmarked for a follow-up assessment at household and child level using the FSVI assessment and CSI assessment tool which was adopted and modified from Uganda OVC Vulnerability Index Tool 2014.

5. At this stage, top 3297 households classified at high and medium risk were earmarked for vulnerability assessment at baseline, these 3297 households accrued from an average number of 300 households in the 11 prevention

\begin{tabular}{|c|c|c|c|c|c|c|c|c|c|}
\hline 3 & Risk Factors & Priority Risk 1 & \begin{tabular}{|c|}
$\begin{array}{c}\text { Priority Risk } \\
\mathbf{2}\end{array}$ \\
\end{tabular} & Priority Risk 3 & $\begin{array}{l}\text { Priority } \\
\text { Risk 4 }\end{array}$ & $\begin{array}{c}\text { Priority Risk } \\
5\end{array}$ & Priority Risk 6 & $\begin{array}{l}\text { Total } \\
\text { Score }\end{array}$ & \\
\hline is & $\begin{array}{l}\text { Assigned Weight (Enter the community } \\
\text { assigned weipht in the respective columns ond } \\
\text { letcommunity score households accordingly) }\end{array}$ & $\begin{array}{r}\text { Povert } \\
\mathbf{5} \\
\end{array}$ & $\begin{array}{l}\text { ERLARENS } \\
\text { RIGHTS } \\
4\end{array}$ & $\begin{array}{l}\text { DomeSTC } \\
\text { NIOLENCE } \\
3\end{array}$ & $\begin{array}{c}\text { Coxprand } \\
\text { tora } \\
2\end{array}$ & $\begin{array}{l}\text { SACE } \\
\text { BEER } \\
\text { GRQP P }\end{array}$ & $\begin{array}{l}\text { Arunkard } \\
\text { ness } \\
0 \\
\end{array}$ & ……... & $\begin{array}{l}\text { Household Ranking } \\
\text { (Low-High Risk) }\end{array}$ \\
\hline SN & Name of Household head & & & & & & & & \\
\hline 1 & EUNICE SIK ELLO & 5 & & 3 & 2 & & & 10 & Hedigh \\
\hline 2 & BEATRESS OMONIA & 5 & & & 2 & & & 7 & Medilen \\
\hline 3 & APIO Eval INE & 5 & & & & & & 5 & mediun \\
\hline 4 & OKELL BEnNaL $D$ & 5 & $=$ & 3 & & & & 8 & Mediūm \\
\hline 5 & OCEPA $155 \mathrm{AC}$ & 5 & 4 & 3 & & 1 & 0 & 13 & \\
\hline 6 & OKELLO EMMANUEZ & 5 & 3 & 3 & & & 0 & 8 & Mediu m. \\
\hline 7 & OJOK IVAN & 5 & & 3 & & 1 & 0 & 9 & Medium. \\
\hline 8 & OWoO IoktN. & 5 & & 3 & 2 & 1 & & 11 & High: \\
\hline 9 & $O K \in L L O \operatorname{Iim} n-1$. & 5 & & & & & & 5 & medivm \\
\hline 10 & SIA ONMANGA & 5 & & 3 & & & 0 & 8 & medium. \\
\hline 11. & BETTY NYANGA & 5 & & 3 & & 1 & & 9 & thredium \\
\hline 12 & BeTtY CAONGO & 5 & & & & & & 5 & medum \\
\hline
\end{tabular}

Fig. 2 Sample of completed ARCHI card 
districts ${ }^{1}$, excluding Wakiso district which implemented only objective 2 of the project. A total of 6754 children within the 3297 prevention households were interviewed; 2364 children were in age group of $0-5,2863$ of them were in the age group of 6-14, and 1534 children were aged between 15 and 17 years. Depending on the number of children in each age within a given household, a household with more than one child in a specific age group, only one representative child was randomly selected for that age group. Three children would be interviewed from households with children in all the three target age groups. If the household has only one child in a specific age group, the single child is interviewed. Where there was no child in a specific age group, no interview was done. The maximum number of children interviewed per household was three and the least was one child, depending on the availability of children in the project-targeted age group; 0-5, 6-14 and 15-17 years.

In the reintegration households ${ }^{2}, 832$ households were assessed on FSVI, 1119 reunified children were assessed on CSI; 334 children in the age group 0-5, 434 children were aged 6-14, and 351 children were in the age group of 15-17 years.

The objectives of assessing both the prevention and reintegration households were to:

- Verify and reaffirm the risk factors that led to or were likely to lead to family-child separation or re-separation.

- Assess and classify the level of vulnerability in the targeted households, in order to identify households at high or medium risk of family-child separation.

- Benchmark on the vulnerability status of the households and children in order to measure project effect by end line

The FSVI tool was administered to both reintegration and prevention household heads, alternatively caregivers or to children in case of child headed households. The tool was administered in three waves of data collection: baseline, midterm, and end line. However, in the integration households, the household assessment was ongoing as resettlement continued.

\section{FSVI Assessment Tool}

The FSVI tool was particularly intended to assess the household level vulnerability based on MoGLSD core program areas (CPA) of interventions: household economic livelihood security (CPA 1), access to basic needs (CPA 2), health and care (CPA 3), psychosocial support and basic care (CPA 5),

\footnotetext{
${ }^{1}$ Prevention districts were districts targeted to implement objective one of DOVCU, to reduce unnecessary separation of children from families. The same nomenclature was used to define the project-targeted households, prevention households sometimes referred to as at-risk households.

${ }^{2}$ Reintegration households were households in which a child or children from CCI remand home or street were reunified.
}

and child protection and legal support (CPA 6). The DOVCU project adopted the Department for International Development (DFIDs) sustainable livelihood path model with modifications of a few parameters to suit the MGLSD CPAs to include the non-economic component under CPA 5 and CPA 6 , which was generalized as a measure of social vulnerability.

The FSVI tool had a total of 33 questions, with each question having response options ranging 1-9. Sometimes in an alphabetical order a-f assigned to particular scale of vulnerability ranging from 0 to a maximum of 4 , each vulnerability scale assigned to a particular response was well defined in each question within the CPA elements. Thus, an interviewer would ask each question, circle the appropriate response option given by respondent, and also circle the corresponding vulnerability scale assigned to that item response option.

The vulnerability index was adopted from the MGLSD OVC vulnerability index and the Household Vulnerability Assessment Tool (HVAT) where zero meant no vulnerability completely, 1 indicated low vulnerability, 2 meant a household was moderately vulnerable, scale of 3 indicated a household is vulnerable, and 4 was equated to critical level of vulnerability, alternatively taken as a worst scenario response option. These indices reflected the different degrees of vulnerability; the higher the index, the more vulnerable the household and the lower the index, the lower the vulnerability levels of the household. Each item response option within the FSVI questionnaire was assigned to depict 5 hierarchical scales of vulnerability as described above. All binary responses were assigned a scale of 0,2 , or 4 , depending on its effects to family-child separation as valued by the FSVI review team. Multi-nominal response options were assigned scales of $0,1,2,3$, or 4 . As a result, many item response options were assigned a scale of 2 than 3, depicting an average (moderate) situation in a household. Cronbach's alpha test on the 33 item response scores corresponding to questions in the FSVI tool yielded reliability coefficient of 0.7417 , meaning $74 \%$ of the variance in the scores were considered a reliable variance, which was above $70 \%$ minimally acceptable reliable variance (Nunnally 1967).

As indicated in Table 3 below, CPA 1: household economic livelihood security was assigned the highest aggregate weight

Table 3 FSVI maximum score attainable at a scale of 4, 3, 2, 1, and 0

\begin{tabular}{lrrrrr}
\hline Core program areas (CPAs) & 4 & 3 & 2 & 1 & 0 \\
\hline CPA 1: Household economic livelihood security & 64 & 12 & 26 & 6 & 0 \\
CPA 2: Access to basic needs & 28 & 9 & 8 & 4 & 0 \\
CPA 3: Health and care & 8 & 0 & 2 & 1 & 0 \\
CPA 5: Psychosocial support and basic care & 12 & 3 & 0 & 3 & 0 \\
CPA 6: Child protection and legal support & 20 & 6 & 4 & 2 & 0 \\
Maximum sum of score attainable across all CPAs & 132 & & & & \\
Minimum sum of score attainable across all CPAs & & & & & 0
\end{tabular}

Source: DOVCU project protocol design 
(sum of scores) given the multiple key elements that the DOVCU project was geared to address under livelihood security: household income, household asset ownership, land use, savings, and household resilience to shocks.

CPA 2: household access to basic needs was assigned the second highest aggregate weight, since the second priority focus of the DOVCU project was to respond to push and pull factors of family-child separation, food security, and child education.

CPA 3: child protection was the third priority area, and the fifth focus area was health (CPA 3) where with the main project target was to create demand/refer participants to the available formal healthcare services, and this specifically targeted households that demonstrated low utilization of healthcare.

Household sum of scores across all CPAs (CPAs 1, 2, 3, 5, and 6) termed as "Risk Analysis" was designed to measure in quantified terms the overall household risk of child separation or re-separation in view of both economic and social protection issues. While sum of score across CPAs 1 and 2 termed as "Economic Destitution Analysis", was premeditated to assess the household economic vulnerability status, these two concepts of vulnerability analysis were adopted and modified from MoGLSD-OVC vulnerability assessment tool.

As indicated in Table 4 below, risk analysis was done by computing total sum of scores across all CPAs (CPAs 1, 2, 3, 5 , and 6); households with total sum of scores above 100 were classified as at high risk, those with total sum of scores ranging from 50 to 99 were classified as at medium risk, and households with total sum of scores below 50 were classified as at low risk of family separation or re-separation.

As indicated in Table 5 below, economic destitution analysis was done by computing total sum of scores across CPA 1 and CPA 2, households with total sum of scores above 49 were classified as destitute households or highly economically venerable households, those with total sum of scores ranging from 25 to 49 were classified as struggling households or moderately economically vulnerable household, and households with total sum of scores below 25 were classified as growing households or household out of critical economic vulnerability. Q-Q plot of the household scores at baseline indicated that the household vulnerability scores were

Table 4 Household risk analysis

\begin{tabular}{ll}
\hline Risk classification & Score ranges $^{\mathrm{a}}$ \\
\hline Low risk & $0-49$ \\
Medium risk & $50-99$ \\
High risk & $100+$ \\
\hline
\end{tabular}

Source: DOVCU project protocol design

${ }^{\mathrm{a}}$ The analysis and categorization of risk levels was based on a summation of total score obtained from CPA 1, CPA 2, CPA 3 , CPA 5, and CPA 6.
Table 5 Household economic destitution analysis

\begin{tabular}{ll}
\hline Economic destitution classification & Score ranges \\
\hline Growing & $0-24$ \\
Struggling & $25-49$ \\
Destitute & $50+$ \\
\hline
\end{tabular}

Source: DOVCU project protocol design

${ }^{\mathrm{a}}$ This analysis and categorization was based on a summation of total score obtained from CPA 1 and CPA 2 (household economic livelihood security and access to basic needs).

normally distributed. In that case, the percentile concept was adopted, scores below 25th percentile was classified as low risk, above 75 th percentile was classified as high risk, and in between 25 th and 75 th percentiles was categorized as medium risk.

Similarly, household economic destitution classification score ranges were determined in appreciation of the percentile concept, the 25th and 75th percentiles of the scores; however, the ranges were slightly adjusted to generate the number of destitute households targeted for cash transfers and the number of moderately economically venerable households targeted with support aimed at improving access to financial credit and capital. The rationale for the non-scientific adjustment was due to the available project budget targeting strictly the destitute and struggling households respectively.

While destitute households were provided with cash transfers, struggling households were targeted with support geared to improve access to financial capital and credit (VSLA associate methodology); all these packages were integrated with financial literacy, micro-enterprise developments skills, business skills, parenting, PSS, and child protection messaging. Househols in the growing class were dropped.

\section{CSI Assessment Tool}

Between one to three children in each of the prevention and reintegrating households were interviewed on the CSI tool, depending on the availability of children in a specific age group. Where there were multiple children in a specific age within the same household, one child would be randomly selected as a representation of all other children in the same age group. During data capture, each child within a household was treated as unit of data point; thus, each household would produce multiple CSI questionnaires depending on the availability of children. In the integrating household, only the reunified child or children were interviewed and the interview processes were ongoing as resettlement continued.

The CSI tool was used to assess the safety status of children $0-5$, children $6-14$, and those aged $15-17$ within households at risk of family-child separation. The tool was also framed to match MoGLSD core program areas 
(CPA) of interventions for OVCs; food and nutrition (CPA 2); health, shelter, and care (CPA 3); education and skills training (CPA 4); psychosocial support (CPA 5); and child protection concern (CPA 6) surrounding the child; the main purpose of the CSI tool was to provide a framework for identifying the needs of children, creating individualized goal-directed service plans and monitoring each age group of children in at risk of separation households.

The CSI tool had a total of 18 questions, with each question having response options ranging from 1 to 5 assigned to particular scale of vulnerability ranging from 0 to a maximum of 4, similar to the FSVI and each vulnerability scale assigned to a particular response was also well defined in each question element within the CPAs of the CSI tool; thus, an interviewer would ask each question, then circle the appropriate response option given by respondent and the corresponding vulnerability scale assigned to that specific response option.

Child Status Index was equally adopted from the MGLSD OVC vulnerability index tool as the FSVI tool and the degree of vulnerability scaling was similar. These indices also reflected the different degrees of vulnerabilities; the higher the index, the more vulnerable the child is and the lower the index, the lower the vulnerability levels of the child.

As indicated in Table 6 below, the highest weight was correspondingly assigned to CPAs 2, 3, and 4 and the second highest weight was assigned to CPA 5; followed by psychosocial status and the least weighted was CPA 6 - child protection concern. Cronbach's alpha test on the 18-item response scores in the CSI tool yielded a reliability coefficient of 0.80 , meaning $80 \%$ of the variance in the scores was considered a reliable variance, which is above $70 \%$ minimally acceptable reliable variance (Nunnally, 1978).

Total sum of scores across all CPAs (CPAs 1, 2, 3, 5, and 6) of the CSI tool was designed to measure in quantified terms, the child vulnerability and their risk to family separation or re-separation. As indicated in Table 7 below, child vulnerability analysis was done by computing the total sum of scores across all CSI CPAs 2, 3, 4, 5, and 6. Where children with total sum of scores above 49 were classified as at critical level

Table 6 CSI score attainable at a scale of $4,3,2$, 1, and 0

\begin{tabular}{lrrrrrr}
\hline \multicolumn{2}{l}{ Core program areas (CPAs) } & 4 & 3 & 2 & 1 & 0 \\
\hline CPA 2: & Food and nutrition & 16 & 12 & 6 & 3 & 0 \\
CPA 3: $\quad$ Health, shelter, and care & 16 & 12 & 8 & 4 & 0 \\
CPA 4: $\quad$ Education and skills training & 16 & 9 & 8 & 3 & 0 \\
CPA 5: $\quad$ Psychosocial support & 16 & 6 & 4 & 4 & 0 \\
CPA 6: $\quad$ Child protection & 8 & 6 & 4 & 2 & 0 \\
Maximum sum of score attainable across all CPAs & 72 & & & & \\
Minimum sum of score attainable across all CPAs & & & & & & 0 \\
\hline
\end{tabular}

Table 7 Child vulnerability analysis

\begin{tabular}{ll}
\hline Vulnerability classification & Score ranges $^{\text {a }}$ \\
\hline Critically vulnerable & 50 and above \\
Medium vulnerability & $20-49$ \\
Low vulnerability & $0-19$ \\
\hline
\end{tabular}

Source: DOVCU project protocol design

${ }^{\mathrm{a}}$ This analysis and categorization of risk levels was based on a summation of total score obtained from CPA 1, CPA 2, CPA 3, CPA 5, and CPA 6.

of vulnerability, children with total sum of scores ranging from 20 to 49 were classified as at medium level of vulnerability, and those with total sum of scores below 20 were classified as at low level of vulnerability; these results gave a basis for case managers and para-social workers to design an individualized goal-directed service plans and monitoring of children in the prevention households. Each child was scored separately since they were treated as a separate data unit even if they were children from the same household; the only similarity is that the children's records were linked to their respective households by the household ID; thus, a household record could be mirrored with their data of children in the age group 0-5, 6-14, and 15-17.

A bell-shaped curve on the histogram of the frequency distribution indicated normality in the child vulnerability scores. Approximately, child vulnerability scores below 25th percentile were categorized as at low vulnerability, above 75 th percentile were classified as at critical vulnerability, and in between 25 th and 75 th percentiles were categorized as at medium level of vulnerability.

Cronbach's alpha test on the five composite items (total CPA scores; CPAs 2, 3, 4, 5, and 6) yielded scale reliability coefficient of 0.74 , meaning $74 \%$ of the variance in the composite scores across CSI CPAs was considered a reliable variance.

\section{Conclusions and Recommendations}

In recent years, different approaches have been suggested in understanding community perspective on issues related to children and their families. UNICEF (2005) designed the Children Friendly Space (CFS) approach that was used to engage the community in refugee camps discussing issues affecting children in emergency settings. In Uganda, ChildFund in 2008, by then called Christian Children's Fund used the CFS approach to conduct needs assessments for children in camps in order to support the displaced families and their children. Other approaches that have been used include community mapping where on the contrary, the DOVCU applied both qualitative and quantitative approaches but in an 
elaborate systematic sequenced approach of identifying communities that supplied children to child care institutions (CCIs). Qualitatively, using child information from the CCIs about the origin of the children, the supply communities were identified and known. These were then targeted to get an indepth understanding of the factors pushing or pulling children to institutional care.

At community level, engagement of the community members through the PRAs processes gave the community perspective of the push and pull factors that leads to childfamily separation. Through consensus building with community members in PRA workshops, households at risk of family separation were identified. This inclusive process reduced on the selection bias in most cases where the local leader would biasedly select families to benefit from project intervention. One major limitation was that the weight of the PRA scores was very general in nature "community context" and could not depict the magnitude of the risk factor to individual families. For example, a family could have been identified with one major risk factor of child-family separation based on the community PRA rating, hence being classified at low risk of family separation based on the sum of PRA scores; thus, the households would be dropped, hence, denied a chance to be verified at household vulnerability assessment.

On the other hand, the criteria reduced the selection bias by the community leaders; thus, DOVCU participants' identification protocol was very helpful in targeting communities, household, and children with highest risk of separation. The systematic process also guided the field team in engaging with the necessary key stakeholders in the project processes, and it also facilitated the buy in from the stakeholders. The consensus building workshop and feedback meetings given at the districts and sub-counties level were very important in ensuring ownership of the project targeting process. In a few instances where the results were not commensurate enough to the facts on ground based on district knowledge, the project triangulated the district opinion in decision-making process, especially in the selection of the parishes of target.

Quantitative approaches of using evidence from the results at various levels of analysis, desk reviews, CCI assessment results, CCI child data results, PRA results, and household and child level assessment complemented each other, thus enriching the targeting methodology. For example, CCI child data on parental status and child willingness to be reunified provided the first step in the case management processes: family tracing, child preparation, family preparation, and engagement of the district authority on the resettlement. Information on the circumstances that drives children into institutional care directed the project staff on the design and development of case plans for each child as they are prepared children for reunification.

Worth noting is the challenge of managing children who originated from beyond the 12 project-targeted districts; thus, case management processes of family tracing were an uphill task for the case managers.

The Family Status Vulnerability Index (FSVI) and Child Status Vulnerability (CSI) tools helped the project affirm that the PRA-identified households were at risk and truly vulnerable to family-child separation. Based on the household classifications, right intervention packages were delivered to the households in a commensurate manner. The FSVI assessment results also facilitated the implementation of the livelihood path model where economically vulnerable households were supported with economic empowerment interventions and were anticipated to improve their economic status. Households with high social vulnerability attributes were provided social support services; thus, the FSVI was administered to targeted households at bench marking, midterm review, and at end line to evaluate the project effect on the targeted vulnerable household.

The FSVI tool measured/captured five core program areas as indicated above, the tool was adopted from the MGLSD HVAT tool. Besides the economic vulnerability of the families/households that was measured by CPA 1 and CPA 2, other CPAs pointed out the non-economic/ social vulnerability of the families, given that DOVCU project was designed to deliver an integrated package of services-both economic and social services to the families. Thus, results on the non-economic variables/ parameters pointed out the social vulnerability of the households that later guided the project on the sequencing of social intervention that each family needed. By design, the non-economic interventions would be delivered to the households before the economic interventions, with an idea that these social services integrated with financial literacy would prepare the households to better manage cash, improve their decision-making, and improve parenting among caregivers.

Although the FSVI tool was very helpful in guiding the project in arriving at the different categories of households for support, there were some limitations with the tool. The tool was not comprehensive enough in capturing in-depth the dimensions and indicators of family vulnerability. For example, food and nutrition security lacked robust track of food consumption scores and household dietary diversity; health indicators based on WHO standards, focused on use of formal health services, lacked track of incidences of diseases among the participants; and key education indicators based on UNESCO, access to improved water and sanitation based on the WHO standards, housing conditions, UN HABITAT, protection and violence measures.

The CSI tool was helpful in the ascertaining the vulnerability of children $0-5$ years, 6-14, and 15-17 years of age in targeted households, thus, creating individualized goaldirected service plans and monitoring of children's wellbeing. 
Acknowledgements Financial support for the DOVCU project came from the US Agency for International Development (USAID; Grant No. GH-008) through Displaced Children and Orphans Fund Project grant. We are grateful to the staff at the ChildFund International in Uganda for monitoring the project implementation process. Our special thanks go to all children, their caregiving families, and institutions who agreed to participate in the project.

Funding This study was funded by USAID under Displaced Children and Orphans Fund Project grant (Grant No. GH-008).

\section{Compliance with Ethical Standards}

Verbal consent was obtained from all individual participants included in the study.

Conflict of Interest The authors declare that they have no conflict of interest.

Ethical Approval Data utilized in this paper was collected as part of monitoring and evaluation procedures.

Open Access This article is distributed under the terms of the Creative Commons Attribution 4.0 International License (http:// creativecommons.org/licenses/by/4.0/), which permits unrestricted use, distribution, and reproduction in any medium, provided you give appropriate credit to the original author(s) and the source, provide a link to the Creative Commons license, and indicate if changes were made.

Publisher's Note Springer Nature remains neutral with regard to jurisdictional claims in published maps and institutional affiliations.

\section{References}

Bee H, Boyd D (2004) Developing Child, 10th edition. Pearson, London Better Care Network \& UNICEF. (2009). Manual for measurement of indicators for children in formal care. New York: Better Care Network and UNICEF.

Browne, K. (2009). The risk of harm to young children in institutional care. London: Better Care Network and Save the Children.

Carlson, M., \& Earls, F. (1997). Psychological and neuroendocrinological sequelae of early social deprivation in institutionalized children in Romania. Annual New York Academy of Science., 807(1 Integrative N), 419-428.

Colton, M. (2002). Factors associated with abuse in residential child care institutions. Society and Children, 16(1), 33-44.

Csaky, C. (2009). Keeping children out of harmful institutions: why we should be investing in family-based care. London: Save the Children UK.

De Silva, N., \& Punchihewa, A. G. (2011). Save the Children push and pull factors of institutionalization of children. A Study based in the Eastern Province of Sri Lanka. Retrieved from https://www. researchgate.net/profile/Nirekha_De_Silva3/publication/ 233942527_Push_and_Pull_Factors_of_Institutionalization_of Children_A_Study_Based_in_the_Eastern_Province_of_Sri Lanka/links/0912f50d2ad0a696a5000000/Pull-Factors-ofInstitutionalization-of-Children-A-Study-Based-in-the-EasternProvince-of-Sri-Lanka.pdf.

Galappatti, A. (2002). Caring for separated children: An approach for Eastern Sri Lanka: Save the children Norway, October 2002. https://resourcecentre.savethechildren.net/library/caring-separatedchildren-approach-eastern-sri-lanka.

Government of Uganda. (2012). Uganda Alternative Care framework 2012. Kampala: Ministry of Gender Labour and Social Development.

Guinnar, M. R., \& Van Dulmen, M. H. M. (2007). Behavior problems in postinstitutionalized internationally adopted children. Development and Psychopathology. Retrieved from https://doi.org/10.1017/ S0954579407070071.

Johnson, D. E., \& Gunnar, M. R. (2011). Growth failure in institutionalized children. Monographs of the Society for Research in Child Development, 76(4), 92-126. https://doi.org/10.1111/j.1540-5834. 2011.00629.x.

Juffer, F., Bakermans-Kranenburg, M. J., \& van IJzendoorn, M. H. (Eds.). (2007). Monographs in parenting series. Promoting positive parenting: An attachment-based intervention (1st ed.). New York, NY: Taylor \& Francis Group/Lawrence Erlbaum Associates.

Juffer, F., Bakermans-Kranenburg, M. J., \& van IJzendoorn, M. H. (Eds.). (2008). Monographs in parenting series. Promoting positive parenting: An attachment-based intervention. New York, NY: Taylor \& Francis Group/Lawrence Erlbaum Associates.

Kreppner J. M., O'Connor T. G. \& Rutter M. (2001). Can inattention/over activity be an institutional deprivation syndrome? Retrieved from https://www.ncbi.nlm.nih.gov/pubmed/11761285.

MGLSD. (2010). Assessment of the status of babies and children's homes in Uganda. Kampala: Ministry of Gender Labour and Social Development.

MGLSD. (2011). Children (approved home) regulations assessment toolkit. Kampala: Ministry of Gender Labour and Social Development.

MGLSD. (2012). Baseline study on the state of institutional care. Kampala: Ministry of Gender Labour and Social Development.

Moulson, M. C., Westerlund, A., Fox, N. A., Zeanah, C. H., \& Nelson, C. A. (2009). The effects of early experience on face recognition: An event-related potential study of institutionalized children in Romania. Child Development. https://doi.org/10.1111/j.1467-8624. 2009.01315.x.

Nunnally, J. C., Koplin, J. H., Blanton, R. L., \& Shaw, R. E. (1967). Individual differences in word association in relation to pairedassociate learning. Journal of Verbal Learning and Verbal Behavior. https://doi.org/10.1016/S0022-5371(67)80059-8.

Parry-Williams, J. 2006 Suggestions for a strategy to develop alternative care and division systems through government structures in Sri Lanka. Save the Children in Sri Lanka.

Priyangani, U., Bernali Fernando, R., Udayani Jayaweera, A., Kumari, J., Mahalingam, A., Kanagaratnam, J., ... Sabasaraj, T. (2005). Research Project on Children in Institutional Care: The Status of their Rights and Protection in Sri Lanka Final Narrative Report Report Submitted by Save the Children in Sri Lanka and Save the Children Canada, (September). Retrieved from https:// bettercarenetwork.org/sites/default/files/attachments/Children in Institutional Care.pdf.

Roccella, C. (2007). Out of sight, out of mind: report of voluntary residential institutions for children in Sri Lanka- statistical analysis. Sri Lanka: Ministry of Child Development and women's empowerment and UNICEF.

Sellick, C. (1998). The use of institutional care for children across Europe. Europe Journal of Social work, 1(3), 301-310.

Smyke, A. T., Koga, S. F. M., Johnson, D. E., Zeanah, C. H., \& the BEIP Core Group. (2007). The caregiving context in institution reared and family reared infants and toddlers in Romania. Journal of Child Psychology and Psychiatry. Available at http://www. bucharestearlyinterventionproject.org/Smyke_et_al__2007_.pdf.

Tolfree. (1995). Roofs and roots: the care of separated children in the developing world. London: Save the Children UK. 
Toner, D. (2012). Review of for tranquility and order: family and community on Mexico's northern frontier, 1800-1850, by Shelton, L. M. Bulletin of Spanish Studies, 89(1), 155-157 ISSN 1475-3820.

UNICEF. (2009). 2009 UNICEF. State of the world's children 2009. New York: United Nations Children's Fund (UNICEF).

UNICEF. (2010). 2010 UNICEF. At home or in a home? Formal care and adoption of children in Eastern Europe and Central Asia. Geneva: UNICEF Regional office for CEE/CIS.

UNICEF (2015) Situation analysis of children in Uganda, Accessible at: https://www.unicef.org/Uganda/UGPublicAdvocacyBooklet(FINALFINALFORWEB). Accessed 04 Dec 2017

United Nations. (2009). Annex guidelines for the alternative care of children, 53(53), 3. Retrieved from https://doi.org/10.1017/ S002081830001924X.
Walakira, E. J., Ddumba-Nyanzi, I., \& Kaawa-Mafigiri, D. (2014a). HIV and AIDS and its impact on child well-being. In Handbook of Child Well-Being: Theories, Methods and Policies in Global Perspective. Retrieved from https://doi.org/10.1007/978-90-481-9063-8_91.

Walakira, E. J., Ddumba-Nyanzi, I., Lishan, S. \& Baizerman, M. (2014b). No place is safe: violence against and among children and youth in street situations in Uganda, Vulnerable Children and Youth Studies: An International Interdisciplinary. Journal for Research, Policy and Care. Retrieved from https://doi.org/10.1080/17450128.2014. 934750

Zeanah, C. H., Smyke, A. T., Koga, S. F. M., Carlson, E., \& BEIP Core Group. (2005). Attachment in institutionalized and community children in Romania. Child Development, 76(5), 1015-1028 Available at https:/www.ncbi.nlm.nih.gov/pubmed/16149999. 